\title{
Evaluación del estado nutricional según compartimentos somático, graso y proteico-visceral al ingreso en una unidad de cuidados intensivos
}

\author{
María Esther Rodríguez Delgado ${ }^{1}$ Luis Albendín García ${ }^{2}$ y Ginesa López \\ Torres $^{3}$ \\ ${ }^{1}$ Hospital Santa Ana (España); ${ }^{2}$ Dispositivo de Apoyo Córdoba Sur (España); \\ ${ }^{3}$ Dispositivo de Apoyo Granada (España)
}

La alta prevalencia de malnutrición en el ámbito hospitalario continúa siendo un problema de especial relevancia en España en la actualidad debido al impacto negativo sobre la evolución clínica del paciente hospitalizado. Numerosos estudios evidencian tasas de desnutrición hospitalaria cercanas a un 33-50\% de la población hospitalizada. Este impacto es mucho más importante y de mayor trascendencia cuando hablamos de pacientes críticos dentro de la unidad de Cuidados Intensivos. Estados nutricionales y metabólicos deficitarios tienen una repercusión directa sobre el enfermo, entorpeciendo la evolución clínica del enfermo e incrementando las tasas de morbimortalidad y de complicaciones hospitalarias así como los costes sanitarios derivados de ello. Desarrollar un adecuado abordaje nutricional mediante soportes nutricionales es un objetivo fundamental y un pilar básico para un óptimo manejo del paciente críticamente enfermo, pudiéndose ver obstaculizado en numerosas ocasiones, especialmente en pacientes que cursan con hipercatabolismo e hipermetabolismo con un consumo energético importante secundario al proceso patológico que motivó el ingreso. A todo ello hay que sumar la gran dificultad que supone una valoración integral y completa de su estado nutricional donde muchos de estos parámetros, encuestas nutricionales y pruebas funcionales puede resultar complicadas de realizar, poco fidedignos, o pueden aportan datos incorrectos, incompletos o con imposibilidad de su realización. Palabras clave: Malnutrición proteico-calórica, bioquímica, metabolismo, cuidados intensivos.

Nutritional status by somatic sub, fat and protein-visceral at entry in an intensive care unit. The high prevalence of malnutrition in hospitals remains a problem of special importance in Spain at present due to the negative impact on the clinical course of hospitalized patients. Numerous studies show hospital malnutrition rates close to $33-50 \%$ of the hospitalized population. This impact is much more important and of greater importance when it comes to critically ill patients in the intensive care unit. Nutritional and metabolic deficit States have a direct impact on the patient, hindering the clinical evolution of the patient and increasing morbidity and mortality rates and hospital complications and healthcare costs arising. Develop an appropriate nutritional approach through nutritional media is a fundamental goal and a basic pillar for optimal management of the critically ill patient, being able I see hampered in many cases, especially in patients who present with hypercatabolism and hypermetabolism with significant energy consumption secondary to the disease process leading to admission. To this must be added the great difficulty of a comprehensive and complete assessment of nutritional status where many of these parameters, nutritional surveys and functional tests can be complicated to perform, unreliable, or may provide incorrect, incomplete or impossible data its realization.

Keywords: Protein-energy malnutrition, biochemistry, metabolism, intensive care.

Correspondencia: María Esther Rodríguez Delgado. Unidad de Gestión Clínica de Cuidados Críticos y Urgencias. Hospital Santa Ana. Avda. Enrique Martín Cuevas, s/n. Motril, Granada (España). E-mail: esther._rd@hotmail.com 
La alta prevalencia de malnutrición hospitalaria (McWhirter y Pennigton, 1997) tiene una repercusión importante sobre la evolución clínica, las tasas de morbi-mortalidad (Ulibarri, Picón, García y Mancha, 2002) y los costes socio-sanitarios hospitalarios. Estas cifras relacionadas con cualquier grado de déficit nutricional en el ámbito hospitalario continúa siendo un problema de especial relevancia en España en la actualidad debido al impacto negativo sobre la evolución clínica del paciente hospitalizado.

Numerosos estudios evidencia tasas de desnutrición hospitalaria cercanas a un 33-50\% de la población hospitalizada, independientemente del tipo de patología que motivó el ingreso (García, Álvarez, Calvo, Ulíbarri, Del Río, Galbán y García, 2005). Este impacto es mucho más importante y de mayor trascendencia cuando hablamos de pacientes críticos dentro de la Unidad de Cuidados Intensivos.

Desarrollar un adecuado manejo nutricional se ve obstaculizado en pacientes con hipercatabolismo e hipermetabolismo como el caso del paciente crítico (Ortiz, Montejo, Jiménez, Lopez, García, Grau, Acosta, Mesejo, Fernández, Ordoñez, Bonet y Blesa, 2005) donde la respuesta inflamatoria secundaria al estrés unido a un estado deficitario nutricional puede tener un impacto negativo sobre el estado del paciente crítico ya que se disminuye en gran medida la capacidad de respuesta al estrés, existe un retraso en la cicatrización y hace que tenga una evolución enlentecida, incrementando las tasas de complicaciones infecciosas y, por ende, de morbimortalidad, estancia y costes económicos (Bertullo y Olano, 2006).

A todo ello, debemos sumarle la gran dificultad en muchas ocasiones para llevar a cabo una valoración integral adecuada del estado nutricional del paciente crítico a través de la anamnesis, historial clínico, valoración subjetiva global u otros cuestionarios validados de valoración nutricional así como para la valoración objetiva mediante parámetros antropométricos, pruebas funcionales y marcadores bioquímicos específicos nutricionales.

Los parámetros antropométricos como el peso o índice de masa corporal implican la obtención de datos de escasa fiabilidad debido a las alteraciones en la distribución hídrica y los cambios en composición corporal tan variable en el paciente crítico.

La valoración subjetiva global (VSG) permite establecer un diagnóstico de déficit nutricional de forma fiable e iniciar una intervención de forma precoz pero, en muchas ocasiones, presenta grandes limitaciones dentro de la unidad de Cuidados Intensivos debido a las características presentes en este tipo de pacientes ya que el estado de conciencia del paciente puede encontrarse gravemente comprometido por la propia patología que motivó el ingreso, la medicación sedante y/o relajante, intubación endotraqueal y ventilación mecánica invasiva, etc. En otras ocasiones, la encuesta realizada a sus familiares a veces arroja datos erróneos, inconexos o incompletos. 
La realización de pruebas funcionales como la dinamometría, la valoración de la contracción del musculo abductor o la espirometría son pruebas poco fidedignas, precisan de la colaboración del paciente y no siempre se encuentran, especialmente en hospitales de tercer nivel con recursos humanos y materiales limitados.

Por todo ello, y dadas las grandes limitaciones en hospitales de tercer nivel y en las unidades de Cuidados Intensivos, nuestro estudio se llevó a cabo a través de la valoración de los distintos marcadores nutricionales específicos para compartimento corporal para evitar errores diagnósticos incurridos por la propia patología del paciente e incrementar la fiabilidad resultante del análisis de estos datos de laboratorio.

\section{Objetivos}

Primario:

Valorar el estado nutricional y metabólico del paciente críticamente enfermo con soporte nutricional artificial enteral al ingreso en la unidad de Cuidados Intensivos a través de los distintos compartimentos corporales mediante determinación analítica de los diferentes marcadores bioquímicos nutricionales específicos para cada compartimento.

Secundarios:

Conocer las distintas alteraciones de los compartimentos corporales músculoesquelético, graso y proteico-visceral tanto si se encuentran instauradas como si están en fase de instauración por una desnutrición previa al ingreso.

Determinar el grado de afectación de los sistemas orgánicos secundarios a dicho déficit nutricional.

Identificar aquellos pacientes críticos con malnutrición o en riesgo de desnutrición.

\section{MÉTODO}

\section{Participantes}

Se realizó un muestreo no probabilístico de conveniencia donde se incluyeron aquellos pacientes que mantuvieron soporte nutricional enteral como único soporte nutricional para realización de un estudio preliminar sobre la malnutrición preexistente al ingreso de la unidad y limitación del tamaño muestral (en un estudio futuro, actualmente en fase de diseño, se hará extensible a aquellos pacientes con soporte nutricional parenteral total, parenteral periférico, terapia combinada enteral y parenteral e ingesta oral).

Pacientes excluidos: Se excluyeron todos aquellos sujetos que tuvieron nutrición parenteral total, nutrición parenteral periférica, terapia combinada con nutrición enteral y parenteral simultánea y aquellos con ingesta oral, edad inferior a 18 
años y, además, también se descartaron aquellos con datos incompletos o erróneos en su recogida y en los que no fue posible revisión de su historial clínico (fallecimiento, traslado interhospitalario, etc.).

Tamaño muestral: 16 pacientes.

El estudio de llevo a cabo aplicando normativa ética basada en las últimas recomendaciones de la guía en investigación biomédica de la Declaración de Helsinki (Asociación Médica Mundial, 1989).

\section{Instrumentos}

Se recogen marcadores bioquímicos nutricionales determinados tanto en sangre como en orina de 24 horas en el servicio de laboratorio de nuestro hospital de todos aquellos pacientes que ingresan en la unidad y se les prescribió soporte nutricional artificial enteral. Las analíticas se llevaron a cabo los lunes y jueves de cada semana y la extracción se realizó a las 7 horas a.m.

Además, se recogen evaluadores pronósticos y de gravedad específicos para el paciente crítico. El índice de Comorbilidad de Charlson y la supervivencia estimada a los 10 años fueron calculados mediante el Charlson Comorbidity Index (Charlson, Charlson, Peterson, Marinopoulos, Briggs y Hollenberg, 2008), mientras que mortalidad estimada y escala de gravedad se llevaron a cabo según escala SAPS III.

El grado de estrés metabólico al ingreso fue determinado mediante ecuación de Harris Benedict junto a modificadores según la patología en personas enfermas (Harris y Benedict, 1919).

\section{Procedimiento}

Se realiza recogida de variables al ingreso del paciente en la unidad (SAPS III, mortalidad estimada, Índice de Charlson, supervivencia estimada) así como de forma diaria y otras con una frecuencia de 2 veces/semana (determinación analitica de marcadores específicos nutricionales tanto en bioquímica en sangre como en orina). Las analíticas fueron extraidas por personal de enfermería de la unidad mediante extracción sanguínea a las 7 horas a.m. y la orina de 24 horas era recogida y remitida a laboratorio por el personal auxiliar de enfermería.

También se lleva a cabo determinación de peso al ingreso y de forma diaria a las 9 horas a.m. mediante bascula incorporada a la cama, que estaba previamente tarada.

Ámbito de estudio: Unidad de Cuidados Intensivos polivalente de un hospital de tercer nivel, de 8 camas de ocupación máxima. Nuestra unidad tuvo una ocupación anual de 513 pacientes ingresados a lo largo del año 2013.

Período de estudio: El estudio fue llevado a cabo durante un total de 14 meses entre los meses comprendidos de enero de 2013 y febrero de 2014.

Variables recogidas: Las variables incluidas fueron las siguientes: 
Variables sociodemográficas: Edad y sexo.

Motivo de Ingreso en Cuidados Intensivos: Patología médica o quirúrgica.

Grado de estrés metabólico ajustado a la respuesta metabólica a la agresión al ingreso en la unidad (sepsis, traumatismo, quemaduras, cirugía, etc.) y tras determinación analítica según nitrógeno excretado en orina.

Parámetros antropométricos: Peso diario, talla e índice de masa corporal.

Evaluadores pronósticos y de gravedad específicos para el paciente crítico: SAPS III (Philipp, Metnitz, Moreno, Almeida, Jordan, Bauer, Abizanda, Lapichino, Edbrooke y Capuzzo, 2005) y mortalidad estimada.

Evaluadores de Comorbilidad: Índice de Comorbilidad de Charlson (Charlson, Pompei, Ales y MacKenzie, 1987) y Supervivencia estimada a los 10 años (Charlson y Paterson, 2008).

Parámetros analíticos plasmáticos y urinarios: Hemograma (hemoglobina, hematocrito, leucocitos y linfocitos), bioquímicos plasmáticos (sodio, potasio, calcio, fósforo, glucosa, urea, creatinina, GPT, GOT, fosfatasa alcalina, bilirrubina total) y específicos nutricionales (albúmina, prealbúmina, transferrina, colesterol total, linfocitos, urea y creatinina excretada en orina de 24 horas, etc.).

Dichas variables eran recogidas de forma diaria y otras con una frecuencia de 2 veces/semana coincidiendo con la determinación analitica de marcadores especificos nutricionales tanto en bioquímica como en orina.

\section{RESULTADOS}

El total de pacientes estudiados (16 pacientes en total) tuvieron una estancia total en UCI de 179 días, con una estancia media de 11,18 días por paciente, con una estancia máxima de 61 días y mínima de 1 día respectivamente.

Del total de pacientes, 11 de ellos $(68.75 \%)$ ingresaron de forma urgente en la unidad e ingresaron en la unidad procedentes de su domicilio a través del servicio de urgencias hospitalario o de los sistemas de emergencias extra hospitalario y, un $31.25 \%$ provenían de planta de hospitalización.

Del total de sujetos, un $87.5 \%$ eran varones y solo un $12.5 \%$ eran de sexo femenino. La edad media de la población estudia era de 67.14 años con una edad máxima de 79 años y mínima de 28 años (Tabla 1).

Tabla 1. Distribución sociodemográfica de la población estudiada

\begin{tabular}{|c|c|c|c|c|c|}
\hline \multirow{2}{*}{ Sexo } & Hombre & $87.50 \%$ & \multirow{2}{*}{ Origen } & Domicilio & $68.75 \%$ \\
\hline & Mujer & $12.50 \%$ & & Planta & $31.25 \%$ \\
\hline \multirow{2}{*}{ Patología } & Médica & $81.25 \%$ & \multirow{2}{*}{ Edad (años) } & Media & $\frac{51.25}{67.14}$ \\
\hline & Ouirúrgica & $18.75 \%$ & & Máx / Mín. & $79 / 28$ \\
\hline
\end{tabular}

Fuente: Elaboración propia 
El SAPS III Medio fue de 62.81 con una Mortalidad estimada media de $44.37 \%$ en la unidad. El índice de Comorbilidad tuvo un valor promedio de 5.28 y un $29.81 \%$ de supervivencia estimada a $\operatorname{los} 10$ años.

En cuanto a la patología que motivó el ingreso en la unidad de Cuidados Intensivos, fue mayoritariamente de tipo médico con un $81.25 \%$ frente a un $18.75 \%$ por motivos quirúrgicos (Tabla 2).

Tabla 2. Valores medios evaluadores pronósticos, de gravedad y comorbilidad de la muestra estudiada

\begin{tabular}{lr}
\hline \multicolumn{2}{l}{ Valores medios de los evaluadores pronósticos, de gravedad y comorbilidad } \\
\hline SAPS III & 62.81 \\
\hline Mortalidad estimada & $44.37 \%$ \\
\hline Índice Comorbilidad Charlson & 5.28 \\
\hline Supervivencia estimada a 10 años & $29.81 \%$ \\
\hline Fuente: Elaboración propia &
\end{tabular}

El grado de estrés metabólico ajustado a la patología al ingreso determinó que un $37.5 \%$ de los pacientes presentaban un grado de estrés intenso, un 37.5\% mostraban estrés moderado: $37.5 \%$ y un $18.75 \%$ era de carácter leve. En tan solo un $6.25 \%$ de la muestra estudiada no había estrés metabólico (Gráfico 1).

Gráfico 1. Grado de estrés metabólico al ingreso en Cuidados Intensivos

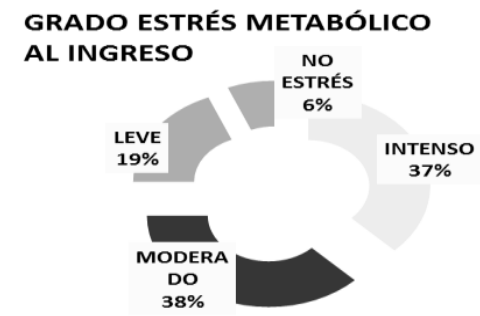

Fuente: Elaboración propia

La valoración de estado nutricional que presentaban al ingreso de la unidad se realizó mediante el análisis de marcadores de laboratorio bioquímicos específicos nutricionales determinados tanto en plasma como en orina recogida en 24 horas.

Para la detección de los déficits nutricionales ya instaurados en el momento del ingreso como aquellos déficits que se encontraban en fase de evolución hacia algún estadio de malnutrición y evitar que pasaran desapercibidos cualquier tipo de malnutrición, se decidió recoger y analizar posteriormente 2 determinaciones seriadas de todos ellos, incluyendo todos los datos analíticos incluidos de las dos primeras analíticas generales desde el momento del ingreso, que en el caso de nuestra unidad, se llevan a cabo todos los martes y los jueves de cada semana. 
Para la valoración del grado de afectación del Compartimento somático: Músculo-Esquelético, se llevó a cabo la determinación del Índice creatinina/talla (I.C.A.) como procedimiento fiable de medición de la proteína existente a nivel muscular.

La creatinina es un derivado metabólico de la creatina, que se encuentra aproximadamente en un $98 \%$ en el músculo, por tanto, los valores resultantes de la creatinina excretada por vía urinaria en diuresis recogida durante un periodo de 24 horas mantiene una buena correlación con la masa magra corporal del sujeto.

El cálculo se llevó a cabo según la ecuación que mostramos a continuación (Figura 1):

$$
\text { Figura 1. Ecuación Índice Creatinina/Talla }
$$

$$
\begin{aligned}
& \% \text { Índice } \frac{\text { Creatinina }}{\text { Talla }}=\frac{\text { Creatinina en orina } 24 \text { horas }(\mathrm{mg})}{\text { Creatinina ideal según talla en } 24 \text { horas }(\mathrm{mg})} \times 100 \\
& \text { Fuente: (Gavilan, 2000). }
\end{aligned}
$$

Donde los valores de Creatinina ideal se calcularon según las estimaciones que hizo Blackburn (Chamorro y Molina, 1998) para la creatinina excretada según los diferentes sexos y alturas para cada uno de ellos.

Tras llevar a cabo el análisis de los valores calculados para el índice Creatinina/Talla según la ecuación anteriormente detallada se puso de manifiesto la existencia de una depleción muscular severa y depleción muscular moderada en el $16.21 \%$ y $37.83 \%$ de los pacientes respectivamente. Esta depleción fue de carácter leve en el $8.11 \%$ de ellos. Sin embargo, tan solo un $37.83 \%$ de la población de enfermos críticos ingresados no presentaban ningún tipo de afectación músculo-esquelética a su ingreso en la Unidad de Cuidados Intensivos (Tabla 3).

Tabla 3. Afectación del compartimento somático

\begin{tabular}{lc}
\hline \multicolumn{2}{c}{ Valoración del compartimento músculo-esquelético } \\
\hline No depleción muscular & $37.83 \%$ \\
\hline Deplección leve & $8.11 \%$ \\
\hline Deplección moderada & $37.83 \%$ \\
\hline Deplección severa & $16.21 \%$ \\
\hline Fuente: Elaboración propia &
\end{tabular}

Para la evaluación del estado del Compartimento Proteico-Visceral se utilizaron las proteínas secretoras hepáticas (albúmina, prealbúmina, transferrina y proteína transportadora del retinol) como indicadores del estado nutricional tanto de vida corta como larga. La proteína transportadora del retinol no fue evaluada por no determinarse en el laboratorio de nuestro hospital.

La determinación de todas tiene una gran ventaja puesto que resulta de gran facilidad en su recogida pero precisa de la valoración simultánea de más de un marcador nutricional para evitar incurrir en errores diagnósticos secundarios a alteraciones por la 
propia enfermedad o padecimiento como en el caso de pacientes críticos con insuficiencia renal agua o insuficiencia hepática aguda.

En cuanto a la valoración de los valores de Albúmina como proteína de vida media larga (20 días) se evidenció una prevalencia de desnutrición severa en un $19.56 \%$ y moderada en un $58.69 \%$ de los enfermos, siendo leve en un $15.22 \%$ de ellos. Tan solo en un $4.35 \%$ no existía afectación a su llegada a la unidad (Tablas 4 y 5).

Tabla 4. Valores normales de las proteínas secretoras hepáticas

\begin{tabular}{|c|c|c|}
\hline \multicolumn{3}{|c|}{ Valores normales y grado de afectación según valores de proteínas secretoras hepática } \\
\hline \multirow{4}{*}{ Albúmina (g/dl) } & Normal & $>3.5$ \\
\hline & Leve & $2.8-3.4$ \\
\hline & Moderada & $2.1-2.7$ \\
\hline & Severa & $<2.1$ \\
\hline \multirow{4}{*}{ Prealbúmina $(\mathrm{mg} / \mathrm{dl})$} & Normal & $20-40$ \\
\hline & Leve & $15-17.9$ \\
\hline & Moderada & $10-14.9$ \\
\hline & Severa & $<10$ \\
\hline \multirow{4}{*}{ Transferrina $(\mathrm{mg} / \mathrm{dl})$} & Normal & $>200$ \\
\hline & Leve & $150-200$ \\
\hline & Moderada & $100-149$ \\
\hline & Severa & $<100$ \\
\hline
\end{tabular}

Fuente: Merino (2011).

Tabla 5. Grado de afectación del compartimento proteico-visceral tras evaluación de valores de albúmina plasmática

\begin{tabular}{lc}
\hline \multicolumn{2}{c}{$\begin{array}{c}\text { Valoración del compartimento proteico- visceral al ingreso mediante } \\
\text { valores de albúmina plasmática }\end{array}$} \\
\hline No desnutrición & $4.35 \%$ \\
\hline Desnutrición leve & $15.22 \%$ \\
\hline Desnutrición moderada & $58.69 \%$ \\
\hline Desnutrición severa & $19.56 \%$ \\
\hline Fuente: Elaboración propia &
\end{tabular}

La prealbúmina como indicador precoz de desnutrición estimó que un $16.66 \%$ y $33.33 \%$ tenían una desnutrición severa y moderada respectivamente. Un $33.33 \%$ presentaban valores normales de prealbúmina $\mathrm{y}$, por consiguiente, no presentaban desnutrición. La evaluación de valores de Transferrina determinó una desnutrición moderada en el $50 \%$ de la muestra y de carácter severo en el 23.68\%. Desnutrición leve $5.26 \%$ (Tablas 6 y 7 ).

Tabla 6. Grado de afectación del compartimento proteico-visceral tras evaluación de valores de prealbúmina plasmática

\begin{tabular}{ll}
\hline $\begin{array}{l}\text { Valoración del compartimento proteico- visceral al ingreso mediante } \\
\text { valores de prealbúmina plasmática }\end{array}$ \\
\hline No desnutrición & $33.33 \%$ \\
\hline Desnutrición leve & $16.68 \%$ \\
\hline Desnutrición moderada & $33.33 \%$ \\
\hline Desnutrición severa & $16.66 \%$ \\
\hline Fuente: Elaboración propia &
\end{tabular}


Tabla 7. Grado de afectación del compartimento proteico-visceral tras evaluación de valores de transferrina

\begin{tabular}{lc}
\hline \multicolumn{2}{c}{$\begin{array}{l}\text { Valoración del compartimento proteico- visceral al ingreso mediante } \\
\text { valores de Transferrina }\end{array}$} \\
\hline No desnutrición & $21.06 \%$ \\
\hline Desnutrición leve & $5.26 \%$ \\
\hline Desnutrición moderada & $50.00 \%$ \\
\hline Desnutrición severa & $23.68 \%$ \\
\hline Fuente: Elaboración propia &
\end{tabular}

La valoración del aspecto calórico presente en la desnutrición permitió evaluar el compartimento graso mediante valores normales y anormales del colesterol, ya que se trata de una molécula presente en los depósitos grasos corporales. El compartimento graso evidenció una desnutrición moderada en el $40 \%$ de los pacientes de la unidad al ingreso, severo y leve en el $16 \%$ de la muestra estudiada.

La estimación de los indicadores de funcionalidad orgánica según el estado nutricional del paciente crítico fue otro aspecto importante que también se estudió mediante el análisis de los valores de linfocitos totales, ya que numerosos estudios han evidenciado la correlación entre déficits nutricionales y prevalencias de complicaciones infecciosas secundarias a las alteraciones acontecidas por alteraciones en la inmunidad celular y humoral en estados de malnutrición, lo que conlleva incrementos en la susceptibilidad de éstos a un estado sépticos. El Recuento linfocitario puso de manifiesto una incidencia de desnutrición de tipo leve en el $28.49 \%$ de los enfermos, moderado en $18.43 \%$ de los enfermos. Existió prevalencia de desnutrición severa en un $29 \%$ de los pacientes y grave en $11 \%$ de los enfermos estudiados.

La desnutrición en el momento del ingreso fue prevalente de tipo mixto $(53.33 \%)$ seguido de desnutrición proteica (22.22\%) y desnutrición calórica en un $20 \%$ de los enfermos (Gráficos 2 y 3 ).

Gráfico 2. Evaluación del compartimento graso

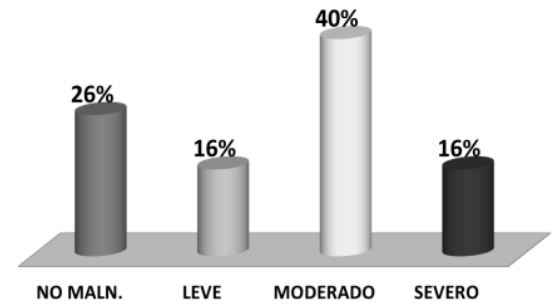

Fuente: Elaboración propia
Grafico 3. Evaluación de la funcionalidad orgánica según recuento linfocitario

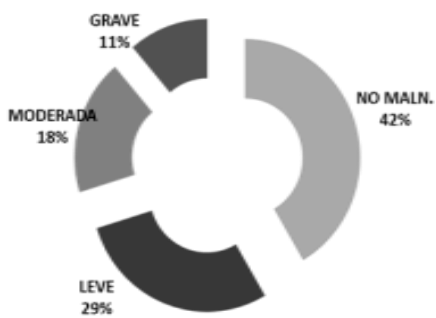




\section{CONCLUSIONES}

La tasa de pacientes con déficit nutricional hospitalario al ingreso de la unidad de Cuidados Intensivos es similar a numerosos estudios realizados de índole internacional. No obstante, esta alta tasa de prevalencia de malnutrición hospitalaria precisa que se adopten medidas a corto-medio plazo a distintos niveles.

Estos niveles deben incluir una intervención precoz desde el momento del ingreso del paciente en el hospital, indistintamente del servicio a su cargo (Planta de hospitalización, unidad de Cuidados Intensivos, etc.) así como mantener una actitud preventiva desde Atención Primaria, especialmente en grupos de riesgo de déficit nutricional como es el caso de ancianos, niños, enfermos crónicos pluripatológicos y de escaso apoyo social, realizando una valoración nutricional periódica así como una adecuada intervención nutricional en colaboración con los servicios de Dietética y Nutrición de referencia.

Una correcta valoración integral permitiría una intervención multidisciplinar precoz y reducir la morbimortalidad, estancia hospitalaria y costes socio-sanitarios, especialmente en pacientes críticos con evoluciones tórpidas secundarias a déficits nutricionales.

\section{REFERENCIAS}

Bertullo, H. y Olano E.(2006). Evaluación nutricional en el paciente crítico. En C. Lovesio (Ed.), Medicina Intensiva. Buenos Aires: El Ateneo. Recuperada en: http://www.intramed.net/sitios/libro_virtual3/pdf/10_1.pdf.

Chamorro, J. y Molina, J.B. (1998). Valoración del estado nutricional. Seminario Médico, 50(3), 87-94.

Charlson, M.E., Charlson, R.E., Paterson, J.C. Marinopoulos, S.S., Briggs, W.M. y Hollenberg, J.P. (2008). The Charlson comorbidity index is adapted to predict costs of chronic disease in primare care patients. Journal of Clinical Epidemiology, 61(12), 1234-1240.

Charlson, M.E., Pompei, P., Ales, K.L. y MacKenzie, C.R. (1987). A new method of classifying prognostic comorbidity in longitudinal studies: development and validation. Journal of Chronic Diseases, 40(5), 373-383.

García, A., Álvarez, J., Calvo, M.V., Ulíbarri, J.L., Del Río, J., Galbán, C. y García, P.P. (2005). Conclusiones del II Foro de Debate SENPE sobre desnutrición hospitalaria. Nutrición Hospitalaria, 20(2), 82-87.

Gavilan, I.(2000). Valoración del estado nutricional y conceptos de desnutrición. En G. Olveira (Ed.), Manual de nutrición clínica (pp. 77-78). Madrid: Díaz de Santos.

McWhirter J. y Pennigton C.R. (1994). Incidence and recognition of malnutrition in hospital. British Medical Journal, 308, 945-948.

Merino, M.J. (2011). Contribución del laboratorio a la valoración del estado nutricional. Recuperada 15 de $\quad$ Septiembre, 2013, de: http //www.moliner.san.gva.es/castellano/Docencia/LAB-VALORACION-NUTRICIONAL.pdf.

Ortiz, C, Montejo, J.C., Jiménez, F., Lopez, J., García de Lorenzo, A., Grau, T., Acosta, J., Mesejo, A., Fernandez, F., Ordoñez, F.J., Bonet, A. y Blesa, A. Grupo de Trabajo de 
Metabolismo y Nutrición de la SEMICYUC (2005). Recomendaciones para la valoración nutricional y el soporte nutricional especializado de los pacientes críticos. Nutrición Hospitalaria, 20(2).

Philipp, G., Metnitz, H., Moreno, P., Almeida, E., Jordan, B., Bauer, P., Abizanda, R., Lapichino, G., Edbrooke, D. y Capuzzo, M., Le Gall on behalf of the SAPS 3 Investigators SAPS-3. (2005). From evaluation of the patient to evaluation of the intensive care unit. Part 1: Objectives, methods and cohort description. Intensive Care Med, 31, 1336-1344.

Ulibarri, J.I., Picón, M.J., García, E. y Mancha, A. (2002). Detección precoz y control de la desnutrición hospitalaria. Nutrición Hospitalaria, 17(3), 139-146.

Recibido: 26 de abril de 2014

Recepción Modificaciones: 26 de mayo de 2014

Aceptado: 15 de agosto de 2014 